\title{
Sustainable, Electrically-conductive Bioepoxy Nanocomposites
}

Daniel Varghai ${ }^{1}$, Anthony Maiorana ${ }^{3}$, Qingkai Meng ${ }^{2}$, Richard A. Gross ${ }^{3}$, and Ica Manas-Zloczower ${ }^{2 *}$,

${ }^{1}$ Biomedical Engineering, Case Western Reserve University, 10900 Euclid Ave, Cleveland, OH 44106

${ }^{2}$ Department of Macromolecular Science and Engineering, Case Western Reserve University, 10900 Euclid Ave, Cleveland, OH 44106

${ }^{3}$ Department of Chemistry and Chemical Biology, Rensselaer Polytechnic Institute, $1108^{\text {th }}$ Street, Troy NY 12180

*Corresponding author: Ica Manas-Zloczower Email: ixm@case.edu; Phone: (216) 368-3596; Address: Kent Hale Smith

Building, Room 515, 2100 Adelbert Rd, Cleveland, OH 44106

Abstract: Biobased epoxy nano-composites composed of untreated multi-wall-carbon-nanotubes (MWCNT) and diphenolic acid-derived biobased epoxy: diglycidyl ether of diphenolate $n$-butyl ester (DGEDP-Bu), were fabricated. Electrical, rheological, and mechanical percolation thresholds were compared between biobased and commercial bisphenol A (DGEBA) epoxy composites. For both epoxies, nanocomposites loaded with $0.05-0.2$ wt \% MWCNT's exhibited electrical and rheological percolation at $0.05 \mathrm{wt} \%$ and $0.2 \mathrm{wt} \%$ respectively. DMA and tensile results revealed that DGEDP-Bu composites exhibited equivalent or superior properties to DGEBA composites. With 0.2 wt $\%$ MWCNT's, DGEDP-Bu nanocomposites exhibited $68 \%$ higher electrical conductivity and a three-fold higher rheological yield stress than those made from DGEBA. Rheological characterization corroborated that continuous MWCNT networks are formed within epoxies between 0.1 and $0.2 \mathrm{wt} \%$ MWCNT's. Moreover, upon MWCNT loading, DGEDP-Bu demonstrates equal mechanical performance and better electrical conductivity than DGEBA.

Keywords: Biobased epoxy, carbon nanotubes, percolation, electrical conductivity, nanocomposite, dispersion. 


\section{INTRODUCTION}

Environmental concerns in the last several decades have exacerbated the need for sustainable materials with the capacity to replace existing petroleum derived standards. This need is particularly pressing in the automotive, wind energy, aerospace and microelectronics industries where petroleum derived epoxy resins are abundantly used due to their superior thermal and mechanical properties, dimensional stability, chemical resistance, electrical insulation, and good wettability with substrates for composite fillers and structural adhesives.[1] These industries have driven global epoxy production to 2 million tons in 2010 and over 20 billion dollars annually.[2] The diglycidal ether of bisphenol A (DGEBA) makes up the vast majority ( 90\%) of all epoxies produced.[2] In addition to being petroleum derived, bisphenol A is a known endocrine disruptor in humans and has been linked to decreased fertility, cancer, and morbidity.[3] A biobased alternative to bisphenol-A that can be utilized as a dropin for epoxy resin production would be of significant relevance to the development of safe, sustainable technologies and energy sources for present and future industries.

Of the current biobased epoxies reported in literature, many are unsuitable for composite fabrication as a result of solidity at $25^{\circ} \mathrm{C}$, poor mechanical properties, or complex synthesis procedures.[2,4] These characteristics make existing biobased epoxy materials unfavorable relative to those from DGEBA, the industry standard, for composite fabrication. However, Maiorana et al. recently reported the synthesis of diglycidyl ether diphenolate $n$-alkyl esters (DGEDP).[4] This new family of biobased epoxy thermoset resins has rheological and thermal properties that can be tailored based on the $n$-alkyl side chain length.[4] DGEDP is derived from levulinic acid, a highly anticipated biobased platform chemical that is reaching demonstration plant levels by GFBiochemicals in Italy.[5] The drop-in potential and sustainability of DGEDP makes it amenable for nanocomposite preparation, where matrix viscosity and processing parameters dictate the final properties. However, there has been no previously reported use of DGEDP for composite synthesis. In this study, we have employed diglycidyl ether of diphenolate $n$ -

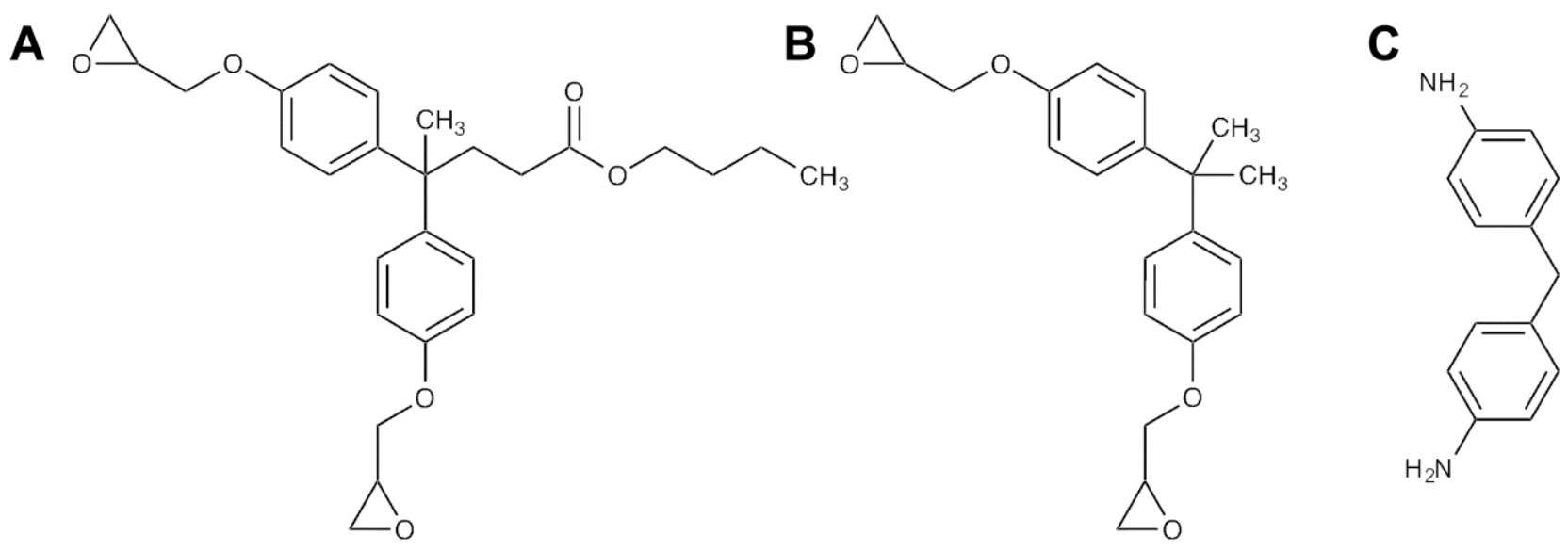

Figure 1

A: Chemical structure of diglycidyl ether of diphenolate $n$-butyl ester (DGEDP-Bu). B: Chemical structure of diglycidal ether of bisphenol A (DGEBA). C. Chemical structure of aromatic hardener diaminodiphenylmethane (DDM). 
butyl ester (DGEDP-Bu) as the resin of choice.

Carbon nanotube (CNT) epoxy nanocomposites have been extensively characterized in literature.[6] The exceptional intrinsic properties of CNTs and high aspect ratios, corresponding to low theoretical percolation thresholds,[7] have generated significant interest towards CNT composite fabrication. An understanding of these dispersed systems can often be obtained through application of percolation theory.[8-10] Statistical percolation theory describes the point at which randomly oriented and dispersed fillers form an infinite lattice in an infinite matrix.[10] Many types of percolation have been examined in CNT composites although much of the literature focuses on electrical, rheological, and mechanical percolation.[7,8,11-21]

Percolation thresholds and dispersion states of CNT's in epoxy matrices are a function of composite processing parameters. Curing temperature, settling time, filler morphology, matrix viscosity and induced shear forces during both the initial disentanglement of CNT's and the addition of hardener, have all been identified as critical to the dispersion state. [7,8,14-16,18,20,22-24] However, the effects of dispersion have varying and opposing influences on different types of percolating networks. Electrical percolation threshold in an insulating matrix, defined as the filler concentration at which continuous conduction pathways cause a dramatic increase in conductivity, is reduced in composites with high levels of CNT aggregation.[7,8,15,18,20,23-25] This trend is in direct contrast with rheological percolation thresholds where uniform filler dispersion is preferential due to the reduced molecular mobility and the increased solid like response of physically hindered individual filler particles. [14,16,21,23,26]

Limitations of statistical percolation theory to predict the characteristics of flocculated networks and agglomerated bundles of filler has led to large discrepancies between theoretical and experimental percolation thresholds.[6] For electrical percolation using CNT's, it is widely accepted that this is primarily due to the dependence of electron conduction on the phenomenon of quantum tunneling. Modeling of these effects has defined the minimum distance required for electron conduction to occur-between 1.8 to $5 \mathrm{~nm} .[13,14]$ These values imply that an electrically percolating network relies on individual CNT's being in close proximity to one another whereas load transfer in mechanically percolating networks requires physical contact. These principles explain the consistently lower electrical percolation thresholds reported relative to other forms of network formation and percolation.[14,26]

Ultimately, the final dispersion state of CNT's is the product of several distinct filler properties. The innately high surface to mass ratios of CNTs leads to morphologies dictated by small scale intermolecular and van der Waals forces. Often, these forces are attractive at interfacial boundaries leading to extensive CNT flocculation.[7] In a well dispersed matrix, however, stability of the dispersion can be achieved by a balance of attractive and electrostatic repulsive forces between individual CNTs.[7] Increased particle mobility that results at relatively higher curing temperatures was furthermore found to induce re-aggregation. Application of low shear rates or high curing temperature can additionally induce agglomeration by 
providing the particles with sufficient kinetic energy to overcome repulsive interactions. In contrast, high shear forces disrupt aggregates and enhances particle dispersion. $[7,20]$

Studies of high performance biobased epoxy composites have thus far been limited by the availability of suitable materials. Many have made use of the scarce, commercially available biobased epoxies such as Greenpoxy (sicomin) [27] or Super Sap (Entropy Bio-Resins Co)[28] while others have used epoxidized vegetable oil derived compounds such as epoxidized linseed (ELO), soybean (ESO), and fatty acids (EGO). [29,30] Generally, these composites compromise mechanical properties or thermal stability in exchange for sustainability. ELO inherently exhibits very low glass transition temperatures $\left(\sim 40^{\circ} \mathrm{C}\right)$ and is frequently mixed with DGEBA in order to improve the overall mechanical properties.[2,29] There has been no reported electrical characterization of biobased epoxy nanocomposites and the incorporation of conductive fillers within biobased epoxy matrices has received little attention.

In this study, we combined our knowledge of DGEDP biobased epoxy resins with similar properties to DGEBA with that of CNT dispersions and composites to demonstrate the fabrication of biobased epoxy CNT nanocomposites. This work illustrates how low-loading percolation varies between electrical, rheological, thermal, mechanical, and thermo-mechanical properties for both biobased and petroleum derived epoxy composites. The DGEDP-CNT nanocomposites fabricated demonstrate superior or competitive properties to that of DGEBA-CNT nanocomposites in nearly every type of characterization performed. Hence, we show that DGEDP-biobased epoxy resin composites with CNT can fill the current void for electrically conductive, high performance, biobased, and sustainable nanocomposites.

\section{EXPERIMENTAL}

Materials. Untreated multiwall carbon nanotubes (MWCNT) grown by catalytic chemical vapor deposition were supplied by Nanocyl (NC7000) specified with an average diameter of $9.5 \mathrm{~nm}$, an average length of $1.5 \mu \mathrm{m}$, and a carbon purity of $\sim 90 \%$. Diglycidal ether of bisphenol A (DGEBA) was purchased from Sigma-Aldrich. Diphenolic acid (DPA) >98\% was purchased from TCI America (D1274). The hardener used for all composite fabrication was diaminodiphenylmethane (DDM), an aromatic amine-based hardener supplied by Sigma Aldrich. All chemicals were purchased in the highest available purity and used as received.

Epoxy Synthesis. The procedure for preparation of the diglycidyl ether of diphenolate $n$-butyl ester (DGEDP-Bu) followed exactly that described previously by Maiorana et al.[4] Furthermore, NMR structural analysis of DGEDP-Bu was consistent with that previously reported.[4]

MWCNT/Epoxy dispersion. Nanocyl NC7000 MWCNTs were weighed into vials to make $0.05,0.1$ and 0.2 wt \% composites. Epoxy (DGEDP-Bu, DGEBA, $15 \mathrm{~g}$ ) was added to each vial. Samples were ultrasonicated (Sonics Vibracell VC750, Sonics and 
Materials Inc., USA) for $25 \mathrm{~min}$ (40 min total time with a $5 \mathrm{sec}$ on $3 \mathrm{sec}$ off pulse) at 40\% amplitude. DGEDP-Bu and DGEBA were sonicated at $80^{\circ} \mathrm{C}$ and $57^{\circ} \mathrm{C}$, respectively, to adjust for differences in neat matrix viscosity. A tapered Ti-6Al-4V $3 \mathrm{~mm}$ microtip (Sonics pt. \# 630-0418) was used for ultra-high intensity local energy input for MWCNT disentangling. Nanocyl NC7000s were selected due to their high aspect ratios and superior percolation.

Composite fabrication. Sonicated epoxy-MWCNT samples were heated to $95^{\circ} \mathrm{C}$ in a thermocouple regulated oil bath so that the cross-linking agent, Diaminodiphenylmethane (DDM) pellets with a melting point of $94^{\circ} \mathrm{C}$, could be liquefied and incorporated. Samples were hand mixed with a stainless steel spatula for 5 min after DDM addition. The amount of DDM added per sample was calculated based on the ratio of amine to epoxy equivalent weights. The epoxy equivalent weight (EEW) of DGEDP-Bu (243) was determined by ASTM standard D1652 whereas the EEW of DGEBA (172) was provided by the supplier. The amine equivalent weight (49.565) was provided by the supplier. The ratio of amine to EEW's was multiplied by the net mass of the sample in order to determine the required mass of DDM. Mixed samples were poured while hot into preheated and degassed silicone molds containing negatives for tensile and DMA specimens. The filled molds were again degassed for 5 min and then the samples were cured at $90^{\circ} \mathrm{C}$ for 4 hours followed by $150^{\circ} \mathrm{C}$ for 4 hours.

In order to slow the curing kinetics of the composites, DDM (Figure 1C) was utilized in place of other cross-linking agents due to the property of its aromatic rings to increase steric hindrance and reduce molecular mobility. Extended cure and gelation time have been shown to improve electrical conductivity as a result of a slight agglomeration of MWCNT's prior to matrix gelation.[18] Furthermore, slower kinetics allows for a longer pot life and processing window to fabricate samples at higher temperatures.

Rheological Testing. Rheological data was collected using a TA Instruments ARES-G2 rheometer equipped with a $40 \mathrm{~mm}$ stainless steel parallel plate geometry at a gap of $5 \mathrm{~mm}$. Samples $(\sim 2 \mathrm{~g})$ were taken from the dispersions prior to DDM addition. Rheological data was collected at $58^{\circ} \mathrm{C}$ for all samples due to DGEBA crystallinity at $25^{\circ} \mathrm{C}$. After waiting for temperature equilibrium, a frequency sweep was conducted with initial ramping from 1 to $100 \mathrm{rad} / \mathrm{s}$ followed immediately by a shear sweep with shear rates ramping from $0.1-100 \mathrm{rev} / \mathrm{s}$.

Tensile Testing. Tensile testing was conducted using an Instron 5960 equipped with a $5 \mathrm{kN}$ load cell. Tensile tests were conducted following the ASTM D638 standard on type III molded dog-bone samples with a crosshead speed of $1 \mathrm{~mm} / \mathrm{min}$. Sample preparation was optimized such that no visible defects or bubbles were present in any tested sample. Each sample was polished to uniform dimensions and the final dimensions were recorded at three points and averaged for stress calculations. Five individual specimens per sample were used for tensile testing and the mechanical properties were averaged and reported with the standard deviation.

Electrical Resistivity. Electrical resistivity measurements were conducted using a PROSTAT PRS-801 Resistance System Set 
surface electrical resistivity platform. Three individual specimens were used for each sample and three measurements were taken in three distinct locations per specimen on untested dual cantilever DMA sample bars. All measurements were averaged and plotted as a function of the wt \% MWCNT loading.

Thermogravimetric Analysis. The thermal stability was assessed using a TA Instruments Q500 thermal gravimetric analyzer using a simple temperature ramp from $25^{\circ} \mathrm{C}$ to $600^{\circ} \mathrm{C}$ at a rate of $10^{\circ} \mathrm{C} / \mathrm{min}$. Each sample tested was a $10 \mathrm{mg}$ section of a cured composite from a $16 \mathrm{~mm}$ dog-bone tensile specimen.

Dynamic Mechanical Analysis. Dynamic mechanical analysis was performed on 40 x 12 x $2 \mathrm{~cm}$ cured rectangular samples with a TA Instruments Q800 ramping from 0 to $200^{\circ} \mathrm{C}$ with an amplitude of $10 \mu \mathrm{m}$ and a frequency of $1 \mathrm{~Hz}$. The glass transition temperature was taken from the peak of the loss modulus.

\section{RESULTS AND DISCUSSION}

\section{Rheology}

Rheological data was collected in order to characterize the dispersion state of MWCNTs in the epoxy matrices and to correlate rheological percolation with electrical and mechanical percolation in cured samples. Huang et al. reported on the inaccuracies of optical techniques to characterize dispersion.[21] They concluded that rheology provides a more consistent and accurate measure of CNT spatial orientation and percolation in polymer systems than the alternative optical methods.[21] Hence, this study uses rheological dispersion data as a proxy for optical characterization.

A shear sweep of the composites loaded at 0.05, 0.1, and $0.2 \mathrm{wt} \%$ MWCNT in both DGEDP-Bu and DGEBA matrices provided information regarding formation of a shear-dependent MWCNT mechanically percolating network. A rheological percolating network is here defined as the filler concentration at which a predominant elastic (solid like) response is observed in an otherwise Newtonian matrix. The neat, unloaded epoxy samples exhibited purely Newtonian behavior with minimal or statistically insignificant differences between zero and infinite shear viscosities. The viscosity of neat DGEDP-Bu is inherently higher than neat DGEBA (0.705 vs. 0.277 Pa.s respectively) which can affect the dynamic percolation of MWCNTs during rheological testing and curing. Due to the importance of matrix viscosity on filler dispersion and aggregation, $[7,18]$ the neat viscosities for DGEDP-Bu and DGEBA were equalized for sonication with MWCNT's. This was achieved by sonicating DGEDP-Bu at $80^{\circ} \mathrm{C}$ and DGEBA $57^{\circ} \mathrm{C}$ in order to equalize the neat viscosity of both systems to a value between $0.2-0.9 \mathrm{~Pa}$.s.

Upon filler loading, a clear rheological percolation is observed at 0.1-0.2 wt \% MWCNT in both epoxies. These values are either below or at the low end of rheological percolation thresholds reported in literature, which mostly range from 0.25-5.0 wt $\%$ MWCNT[14,16,21]. The viscoelastic nature of the loaded composites can be analyzed through procurement of the dispersion yield stress. The yield stress is defined as the stress at which the MWCNT network is temporarily destroyed and the 
force-bearing entanglement of the filler gives way to different morphologies.[16,26,31] Rahatekar et al. proposed that upon application of continued shear, a size reduction of interconnected agglomerates and a much finer non-entangled dispersion state can lead to a yield stress. [32]

The sample's yield stress was obtained by fitting a linearization of the stress-shear flow curves (Figure 2A). The empirical equation describing the parameters of the linear fit originates from the Casson model of viscoelastic dispersions shown in equation $1[26,33]$ :

$$
\sigma^{1 / 2}=\sigma_{\mathrm{c}}^{1 / 2}+\left(\eta_{\mathrm{f}} \dot{\gamma}\right)^{1 / 2}
$$

Plotting $\sigma^{1 / 2}$ against $\dot{\gamma}^{1 / 2}$ where $\sigma$ is the stress and $\gamma$ is the shear rate yields a straight line. Linear regression analysis gives values for the yield stress $\left(\sigma_{\mathrm{c}}\right)$ and infinite shear viscosity. $\left(\eta_{\mathrm{f}}\right)$.

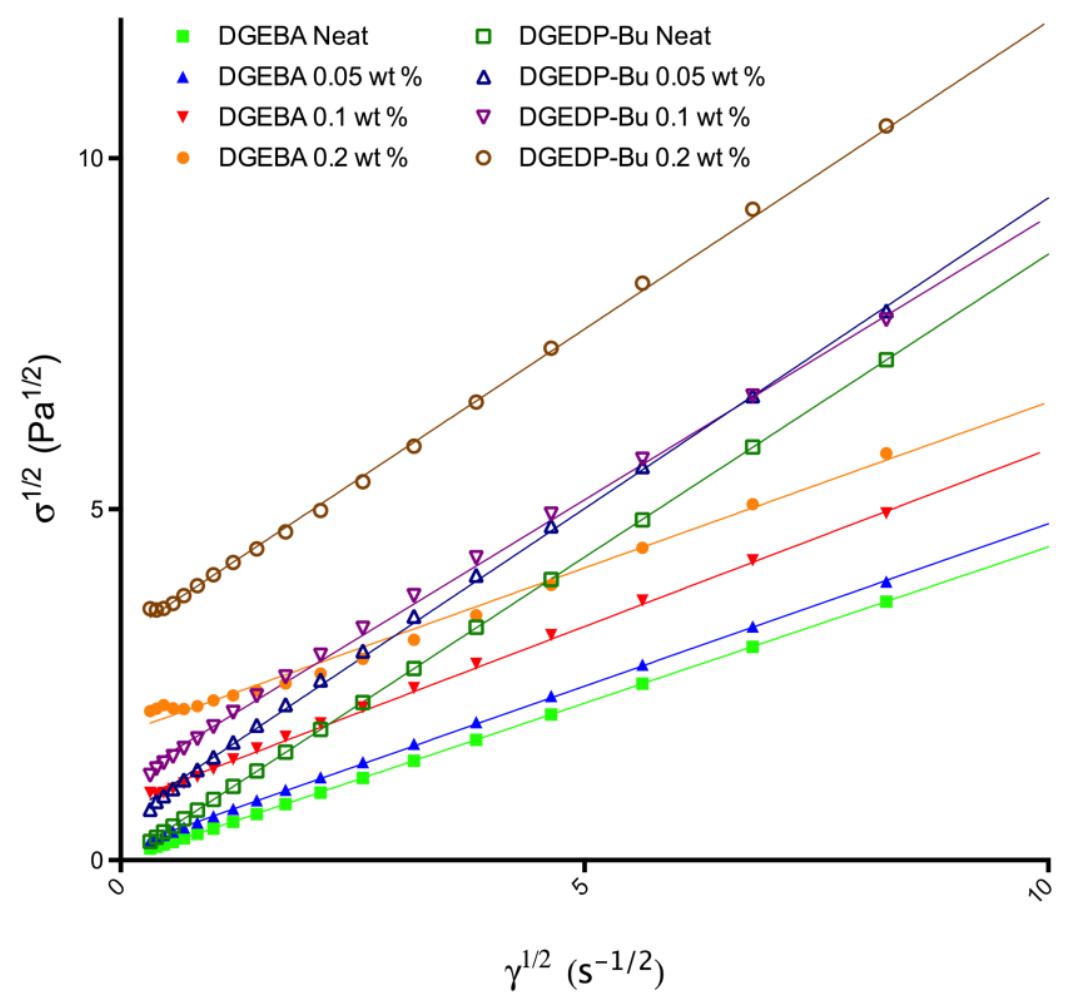

Figure 2

Casson fit of a linearization of the stress vs. shear rate. Closeness of fit is excellent as apparent from visual inspection and reported $R^{2}$ values.

From the tabulated values of the Casson fitted yield stress and viscosity at high shear (Table 1), several important results are apparent. Composites of each epoxy, regardless of the MWCNT loading, demonstrated statistically insignificant variation of infinite shear viscosity. This result is indicative of good processing performance even at high MWCNT loading. Comparing the yield stress of DGEDP-Bu and DGEBA composites, it is apparent that even with similar Newtonian behavior at zero loading, 
there are large variations in yield stress between epoxies with addition of filler. DGEDP-Bu relative to DGEBA exhibits an eleven-fold (1136\%), two-fold (186\%) and three-fold (312\%) increase in $\sigma_{\mathrm{c}}$ at $0.05,0.1$ and $0.2 \mathrm{wt} \%$ MWCNT filler loadings, respectively. These results highlight differences in MWCNT networks formed for each epoxy. MWCNTs in DGEDP-Bu have increased resistance to yield which may imply more structurally supported networks.

\begin{tabular}{|c|c|c|c|}
\hline & $\sigma_{c}$ & $\eta_{f}$ & $R^{2}$ \\
\hline DGEDP-Bu Neat & 0.00 & 0.75 & 1.00 \\
\hline DGEDP-Bu 0.05 wt \% & 0.34 & 0.78 & 1.00 \\
\hline DGEDP-Bu 0.1 wt \% & 1.21 & 0.65 & 1.00 \\
\hline DGEDP-Bu 0.2 wt \% & 10.13 & 0.77 & 1.00 \\
\hline DGEBA Neat & 0.00 & 0.20 & 1.00 \\
\hline DGEBA $0.05 \mathrm{wt} \%$ & 0.03 & 0.21 & 1.00 \\
\hline DGEBA $0.1 \mathrm{wt} \%$ & 0.65 & 0.25 & 1.00 \\
\hline DGEBA $0.2 \mathrm{wt} \%$ & 3.24 & 0.22 & 0.99 \\
\hline
\end{tabular}

Prompted by reports of the influence of settling time on the rheological properties of a dispersion[20,23,25], $0.1 \mathrm{wt} \%$ MWCNT samples were allowed to settle for one week at room temperature following dispersion by ultrasonication. No noticeable differences were observed in rheological properties indicating long term stability of dispersions described herein.

Non-destructive oscillatory inputs were used to measure the dynamic response of the composites. By measuring system response over a wide range of frequencies and the phase between stored energy and viscous dissipation, information on both the elastic and viscous nature of the material was obtained. Because extensive shear is minimized by an oscillating input, a percolating network is not destroyed in the same way as a shear sweep.[16] Rather, a network is gradually subjected to increasing frequencies until the filler network relaxation time exceeds the oscillation period and motion takes place across entanglements unhindered by the presence of nanoparticles.[34] Thus, at high frequencies, the material response is dictated predominantly by the matrix as opposed to the filler. For materials with an elastic component to their response, the less the elastic modulus is frequency dependent, the more fluid-like the material. [14]

Mcclory et al. showed that the percolation state of nanotubes is revealed by the sudden increase in storage modulus or complex viscosity.[14] The point at which storage modulus exceeds loss modulus indicates the transition point from a material dominated by viscous dissipation to a solid like elastic material which can resist flow. Our MWCNT composites demonstrate this transition at $0.1 \mathrm{wt} \%$ indicating the beginning of network formation (Figure 3B, 3C). At $0.2 \mathrm{wt} \%$ MWCNT, composites made from both epoxies demonstrated nearly identical G'-G' behavior with G' > G' for the entire frequency sweep. This 
result can be quantitatively described from the flow behavior index of the Herschel-Bulkley model which describes a non-linear power-law fluid with an added yield stress parameter. [31]

It was previously reported that fitting with the empirical Herschel Bulkley model can describe the non-linear viscoelastic behavior of a dispersion in both steady and oscillatory shear. [16,31,34-37] The fitting equation shown in equation 2 is a version of the Herschel-Bulkley model adapted for oscillatory frequency sweeps[16,31]

$$
\eta^{*}=\frac{\tau_{0}}{\omega}+k \omega^{(n-1)}
$$

where $\eta^{*}$ is the complex viscosity, $\omega$ is the angular frequency, $\tau_{0}$ is the yield stress, $\mathrm{k}$ is the consistency index and $\mathrm{n}$ is the flow behavior index. When $n>1$, the fluid exhibits a shear-thickening behavior; for $n=1$ the fluid has Newtonian behavior; when $n$ $<1$ the fluid shows shear-thinning behavior.

As shown in Figure 3A, the Herschel-Bulkley model fits the data well and the fit parameters are displayed in Table 1. At $0.2 \mathrm{wt} \%$ MWCNT, DGEDP-Bu and DGEBA both have flow behavior indices of 0.6, indicative of a characteristic shear thinning response. At lower loadings than $0.2 \mathrm{wt} \%$, the flow behavior index still indicates shear thinning, but to a lesser extent (Table 1). However, the yield stress corroborates values determined from the Casson fit for rotational rheology. DGEDP-Bu exhibited a $168 \%$ increase in yield stress at $0.2 \mathrm{wt} \%$, and a slightly lower yield stress than that of DGEBA at $0.1 \mathrm{wt} \%$. These results indicate percolation at $0.2 \mathrm{wt} \%$ MWCNT/DGEDP-Bu composites and a clear morphological transition for network formation at $0.2 \mathrm{wt} \%$ MWCNT versus that for corresponding DGEBA composites.

Table 2

Herschel-Bulkley Model-Fit Parameters

\begin{tabular}{lllll}
\hline & $T$ & $k$ & $n$ & $R^{2}$ \\
\hline DGEDP-Bu Neat & 0.0 & 0.81 & 1.0 & 0.257 \\
DGEDP-Bu 0.05 wt \% & 0.2 & 1.75 & 0.9 & 0.983 \\
DGEDP-Bu 0.1 wt \% & 2.0 & 2.42 & 0.8 & 0.999 \\
DGEDP-Bu 0.2 wt \% & 71.6 & 12.84 & 0.6 & 1.000 \\
& & & & \\
DGEBA Neat & 0.0 & 0.17 & 1.1 & 0.290 \\
DGEBA 0.05 wt \% & 0.0 & 0.35 & 0.9 & 0.939 \\
DGEBA 0.1 wt \% & 2.6 & 1.34 & 0.7 & 0.998 \\
DGEBA 0.2 wt \% & 26.7 & 4.31 & 0.6 & 0.999 \\
\hline
\end{tabular}


The differences between MWCNT composites fabricated with DGEDP-Bu and DGEBA are of great interest and relevance to the potential of DGEDP-Bu for replacing DGEBA. One may argue that these differences are attributed to the inherently higher viscosity of DGEDP-Bu which may have promoted greater shear forces during mixing and a higher degree of CNT dispersion. However, the constant matrix viscosity maintained between samples during sonication reduces this possibility. Mcclory et al. has reported that interactions between functionalities on the CNT surface and the matrix are more critical than physical bridging of polymer chains to attaining rheological percolation.[14] Functionalization of CNT's with amine and carboxyl moieties to increase interfacial interaction with the matrix has been reported to increase storage modulus by up to four
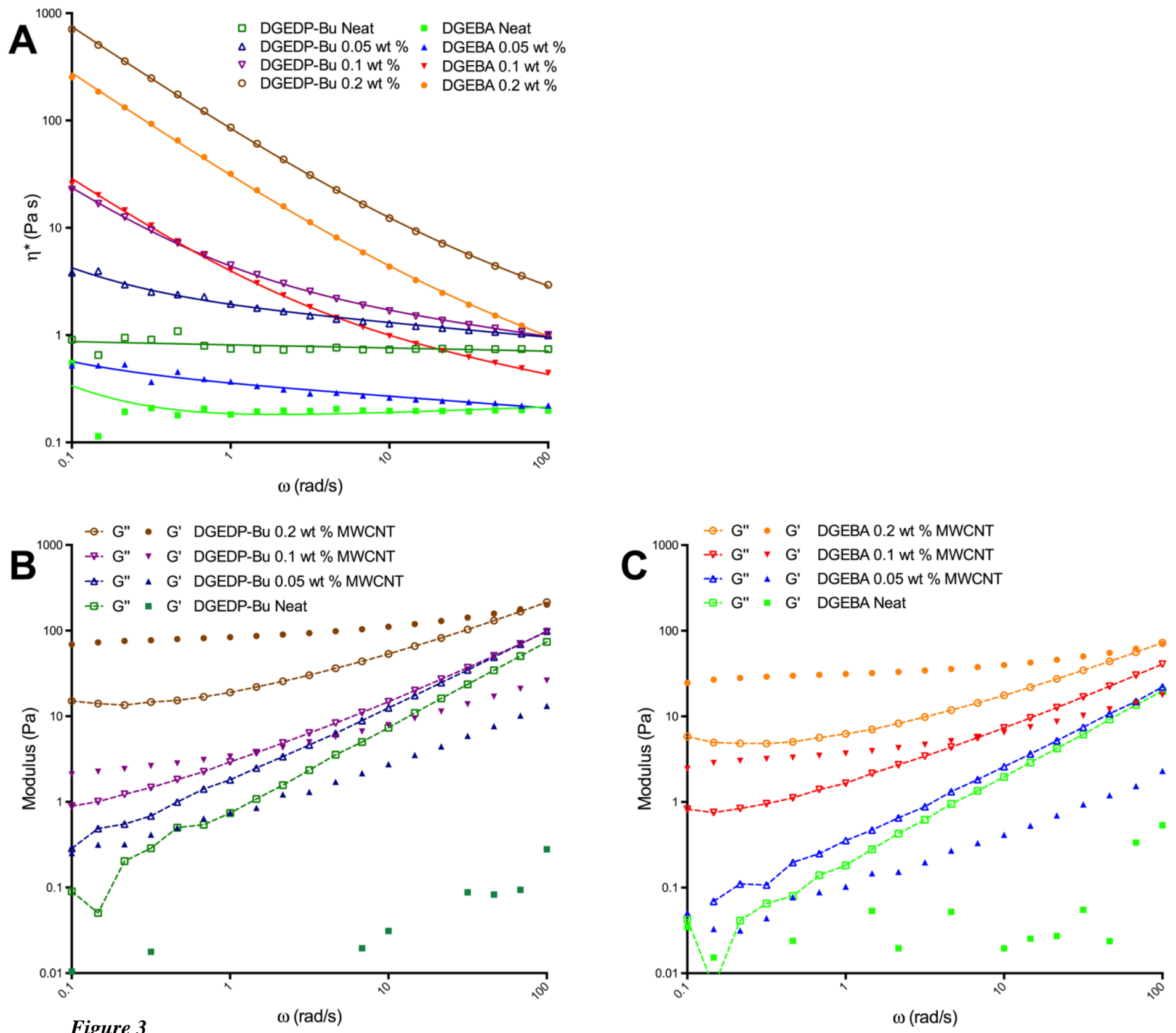

Figure 3

$\omega(\mathrm{rad} / \mathrm{s})$

$\omega(\mathrm{rad} / \mathrm{s})$

A: Complex viscosity as a function of angular frequency. Discrete data points and dashed lines represent experimental data while straight lines are empirical nonlinear regression fits from the Herschel-Bulkley model. Horizontal trends indicate Newtonian behavior while sloped trends are indicative of shear thinning behavior and an increased elastic response (observed with increased MWCNT wt \%). B: Storage modulus ( $G$ ') versus loss modulus (G') of DGEDP-Bu at various MWCNT loadings where $G$ ' $>G$ ' 'indicating the formation of a solid-like elastic network of MWCNT within a Newtonian matrix. C: Storage modulus ( $\left.G^{\prime}\right)$ versus loss modulus ( $\left.G^{\prime \prime}\right)$ of DGEBA at various MWCNT loadings. 
fold and consistently increase $T_{\mathrm{g}}$.[38-41] Therefore, it is possible that DGEDP-Bu's additional hydrophobic side chain could enhance interfacial interactions with CNTs. Additionally, differences in crosslink densities between epoxies due to a discrepancy in the free volume of the matrices could lead to morphological variations in the dispersion. This is supported by dynamic mechanical analysis data discussed in a later section.

\section{Tensile properties:}

Tensile tests were performed on all compositions in order to obtain the stiffness and elongation at break of the composites (Table 3). The Young's modulus $(E)$ of composites made with both epoxies demonstrated no statistically significant differences. The trends, however, indicate marginally increased Young's Modulus at MWCNT loadings of 0.05 and $0.1 \mathrm{wt} \%$ for both epoxies. DGEDP-Bu exhibits a mean Young's modulus at its highest point of $1179 \mathrm{MPa}$ with 0.05 wt $\%$ MWCNT whereas DGEBA's highest mean value is 1129 at $0.1 \mathrm{wt} \%$ MWCNT. At $0.05 \mathrm{wt} \%$ MWCNT, the Young's modulus of the DGEDP-Bu composite is 5\% and 6.7\% higher than that of DGEBA and the neat DGEDP-Bu resin, respectively.

Whereas rheological percolation was observed at 0.1-0.2 wt \% MWCNT, mechanical tests do not corroborate these thresholds. For percolation of MWCNT's to effect the tensile properties of a composite, there must be effective load transfer between the matrix and filler material. While the slight improvement in mechanical results for DGEDP-Bu over DGEBA composites is consistent with our hypothesis of a slightly more homogeneous state of dispersion in DGEDP-Bu, the large variability between samples is indicative of inconsistent load transfer and the lack of a mechanically percolating network. The relatively high standard deviations in the tensile data, even within neat unloaded samples, provides evidence for the presence of an inherent specimen-specimen variability due to the composite fabrication procedure.

\section{Table 3}

E: Young's modulus providing a measure of stiffness. An average of 3-4 specimens were measured for each sample. E: Elongation at break for all fabricated composites.

\begin{tabular}{lll}
\hline & $E(\mathrm{MPa})$ & $\varepsilon(\%)$ \\
\hline DGEDP-Bu Neat & $1122 \pm 39$ & $8.2 \pm 1.2$ \\
DGEDP-Bu 0.05 wt \% & $1179 \pm 23$ & $7.3 \pm 1.9$ \\
DGEDP-Bu 0.1 wt \% & $1137 \pm 46$ & $9.6 \pm 0.9$ \\
DGEDP-Bu 0.2 wt \% & $1100 \pm 69$ & $8.4 \pm 1.1$ \\
& & \\
DGEBA Neat & $1099 \pm 27$ & $8.4 \pm 1.1$ \\
DGEBA 0.05 wt \% & $1141 \pm 45$ & $8.2 \pm 2.0$ \\
DGEBA 0.1 wt \% & $1124 \pm 24$ & $8.2 \pm 1.6$ \\
DGEBA 0.2 wt \% & $1122 \pm 43$ & $8.2 \pm 1.6$ \\
\hline
\end{tabular}

Tensile data for elongation at break (Table $3, \varepsilon$ ) presented no statistically significant variation between samples. This data indicates a lack of large agglomerates within composites which can act as stress concentration points and reduce elongation at 
break. [42-44] Tensile strength results (not shown) additionally showed little to no variation between epoxies and across loadings. Although tensile testing data presented here did not clearly demonstrate composite strengthening and percolation, nanofillers can play a significant role in material toughness and resiliance to fatigue.[45] This would be the subject of a future study.

Tensile tests illustrated the discrepancy between rheological, electrical and mechanical percolation that is consistent with other literature reports. According to Martin et. al[16] filler morphology and functionalization plays a large role in this discrepancy. Comparisons between functionalized graphene sheets (FGS) and MWCNT have illustrated the tendency of MWCNT composites to exhibit relatively low rheological and electrical percolation (due to high aspect ratios) at $0.5 \mathrm{wt} \%$ compared to FGS where percolation was not observed even up to $1.5 \mathrm{wt} \%$. However, tensile testing has demonstrated FGS percolation at $0.25 \mathrm{wt} \%$ surpassing the mechanical properties of MWCNT composites even up to $0.75 \mathrm{wt} \%$. This was attributed to the plate-like morphology of FGS that effectively hinder crack propagation as well as surface functionalization that improves the wettability and interfacial adhesion with the matrix. In contrast, MWCNT's do not provide such hindrance or interfacial interactions.

Consequently, rheological and electrical percolation do not necessarily correlate with mechanical percolation since the critical factors of filler-matrix interactions and prevention of crack propagation which dictate mechanical properties do not necessarily control electrical or rheological properties.

\section{Electrical resistivity}

Surface resistivity measurements were collected and averaged at room temperature across cured specimens (three measurements per specimen) and samples (three specimens per sample) in order to probe the effects of MWCNT loading on the electrical properties of the otherwise insulating epoxy matrix.

The electrical percolation threshold of conductive fillers is defined as the filler concentration at which a large decrease in resistivity (several orders of magnitude) is observed and the resistivity falls into the realm of conductive materials $\left(10^{3}-10^{5}\right.$ S.m)[46]. By this definition, conductive percolation like behavior is observed at $0.05 \mathrm{wt} \%$ MWCNT for both DGEDP-Bu and DGEBA composites, indicated by a six order-of-magnitude decrease in resistivity which occurs relative to the neat resins (Figure 4). At 0.2 wt \% MWCNT in DGEDP-Bu, the highest conductivity of all samples studied herein was achieved with a demonstrated resistivity of $1.01 \times 10^{4} \Omega . \mathrm{m}$ (Figure 4). This represents an eight order-of-magnitude decrease in resistivity over neat DGEDP-Bu. Furthermore, the electrical resistivity of the $0.2 \mathrm{wt} \%$ MWCNT DGEDP-Bu composite is $83 \%$ lower than that of the corresponding DGEBA composite. The resistivity of the $0.2 \mathrm{wt} \%$ MWCNT DGEDP-Bu composite corresponds to the $10^{-4} \mathrm{~S} / \mathrm{m}$ conductivity at which a material is classified as conductive. [46] 


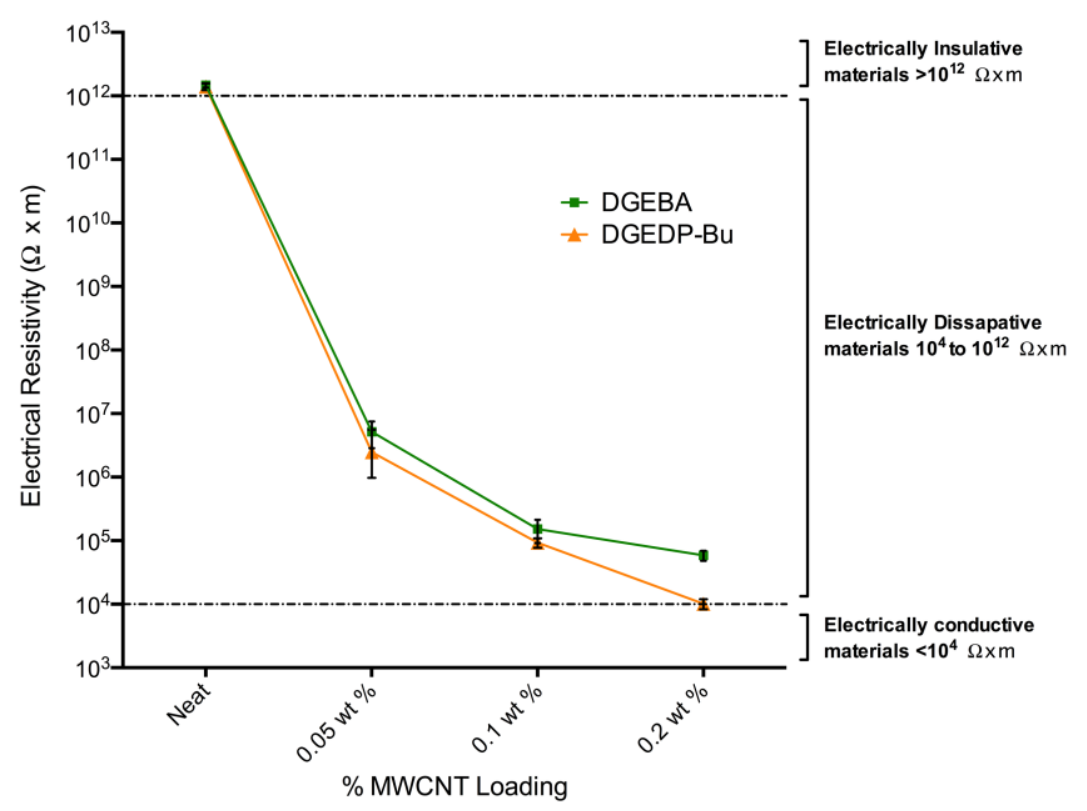

Figure 4

Plot of log of electrical resistivity against MWCNT wt \%. Each line represents a different epoxy matrix. Dotted line indicates resistivity value at which a material is considered conductive, dissipative, and insulative..

Values for the electrical percolation threshold and maximum conductance of MWCNT-epoxy filled systems varies dramatically in existing literature. A review by Bauhofer et al.[6] lists reported percolation thresholds for MWCNT-epoxy systems ranging from $0.0021 \mathrm{wt} \%$ [7] to $5 \mathrm{wt} \%$ [47] and maximum conductivities that range from $1 \times 10^{-5} \mathrm{~S} / \mathrm{m}$ (at $8 \mathrm{wt} \%$ [47] to $5 \mathrm{~S} / \mathrm{m}$ (at $4 \mathrm{wt} \%$ )[48]. Among published results using MWCNTs with aspect ratios near that of the Nanocyl NC7000 MWCNTs (L/D 157) used in this study, values for percolation range from $0.6 \mathrm{wt} \%$ [49] to $4 \mathrm{wt} \%$.[50]. The electrical conductivities of composites developed herein fall well within the range of reported values and exhibit percolation thresholds 91\% below the lowest published value using MWCNTs with similar aspect ratios.

Discrepancies between electrical, rheological and mechanical percolation is attributed to the different requirements of each type of percolating network. The lower loadings reported for electrical percolation versus rheological percolation are explained by the dependence of electrical conduction in CNT networks on quantum tunneling. Simulations by Xiaomei et al. have illustrated that peak conductivity occurs at a distance of $2.5 \AA$ while shorter distances may lead to decreased conductance due to $\mathrm{sp}^{3}$ hybridization and localization of electrons on the CNT surface.[13] At larger inter-particle separation, conductance decreases as the potential barrier becomes wider, diminishing the tunneling probability. The dependence of conduction on a minimum distance between dispersed fillers in a matrix contrasts with requirements to attain ideal mechanical percolating networks where load transfer is important. Ultimately, interfacial distances between fillers, ideal for quantum tunneling of electrons, may not be sufficient to achieve mechanically percolating networks where load transfer is required. 


\section{Thermogravimetric Analysis:}

Thermogravimetric data (Figure 5, Table 4) demonstrated little to no loss in thermal stability upon addition of MWCNT into the epoxy matrices. DGEDP-Bu exhibited similar thermal stability to DGEBA and consistently exhibited a maximum degradation rate (peak of the weight loss derivative curve) at higher temperatures than DGEBA. Overall, the biobased composites have comparable or superior thermal stability to the industry standard DGEBA.

Table 4

Thermal Gravimetric Analysis determined temperatures at which $95 \%$ weight and the maximum degradation rate.

\begin{tabular}{lll}
\hline & $\begin{array}{l}{ }^{\circ} \mathrm{C} \text { at } 95 \% \\
\text { wt. }\end{array}$ & $\begin{array}{l}{ }^{a} \mathrm{C} \text { at maximum } \\
\text { degradation rate }\end{array}$ \\
\hline DGEDP-Bu Neat & 363.3 & 340.0 \\
DGEDP-Bu 0.05 wt \% & 364.7 & 343.0 \\
DGEDP-Bu 0.1 wt \% & 362.3 & 339.3 \\
DGEDP-Bu 0.2 wt \% & 364.7 & 341.0 \\
& & \\
DGEBA Neat & 357.9 & 343.4 \\
DGEBA 0.05 wt \% & 360.8 & 345.3 \\
DGEBA 0.1 wt \% & 357.9 & 339.2 \\
DGEBA 0.2 wt \% & 358.9 & 341.1 \\
\hline
\end{tabular}

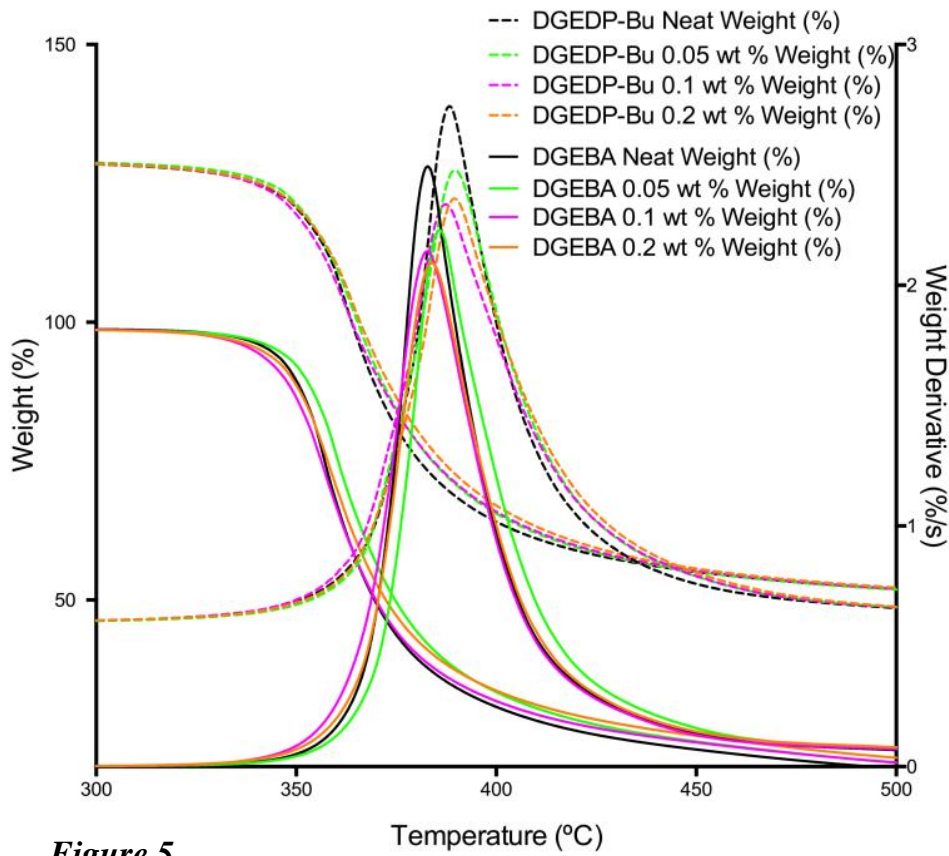

Figure 5

Temperature $\left({ }^{\circ} \mathrm{C}\right)$

Thermal Gravimetric Analysis weight loss (in \%) and weight loss derivative curves $(\% / \mathrm{s})$. Peak of derivative corresponds to the temperature at which the maximum rate of degradation occurs. *Note: DGEDP-Bu curves have been shifted upwards for clarity (weight: $y+30 \%$, weight derivative: $y+0.6 \% / s$ )

\section{Dynamic Mechanical Analysis}

DMA analysis of cured samples provided information regarding the alpha transition temperatures (related to $T_{\mathrm{g}}$ ), taken 
from the peak of the tan delta curve, and the storage moduli of cured samples at various temperatures.

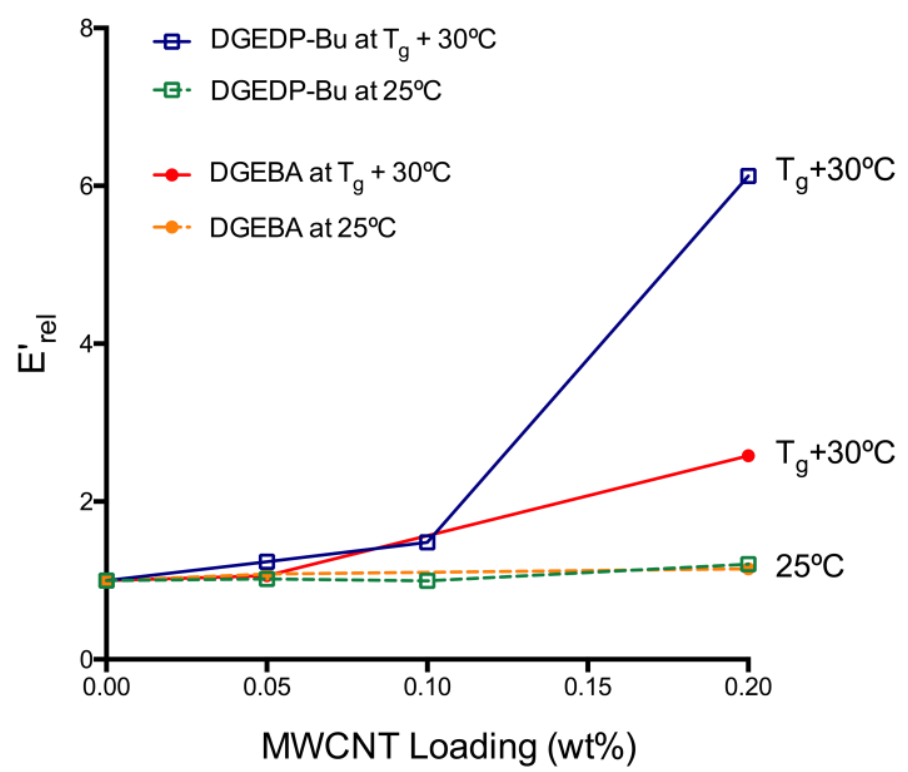

Figure 6

Relative storage modulus from dynamic mechanical analysis for DGEBA and DGEDP-Bu at different temperatures as a function of MWCNT loading fraction. ${ }^{\prime}{ }_{\text {rel }}$ represents the relative storage modulus of the composite with respect to the neat resin. $E_{r e l}^{\prime}$ values are found at $25^{\circ} \mathrm{C}$ to illustrate the glassy-like modulus and $30^{\circ} \mathrm{C}+\mathrm{T}_{\mathrm{g}}$ for the rubber like phase of the composite.

Table 5

Dynamic Mechanical Analysis measurements. $G^{\prime}$ is determined at $25^{\circ} \mathrm{C}$. *Note: DGEBA 0.1 wt \% MWCNT was not included as the sample was unrecoverable.

\begin{tabular}{lll}
\hline & $G^{\prime}(\mathrm{MPa})$ & $T_{g}\left({ }^{\circ} \mathrm{C}\right)$ \\
\hline DGEDP-Bu Neat & $2320 \pm 135$ & $137 \pm 1$ \\
DGEDP-Bu 0.05 wt \% & $2362 \pm 114$ & $135 \pm 2$ \\
DGEDP-Bu 0.1 wt \% & $2311 \pm 96$ & $135 \pm 5$ \\
DGEDP-Bu 0.2 wt \% & $2800 \pm 31$ & $126 \pm 0$ \\
& & \\
DGEBA Neat & $2161 \pm 235$ & $185 \pm 3$ \\
DGEBA 0.05 wt \% & $2334 \pm 43$ & $186 \pm 2$ \\
DGEBA 0.2 wt \% & $2477 \pm 52$ & $184 \pm 1$ \\
\hline
\end{tabular}

DGEDP-Bu exhibits a maximum storage modulus of $2800 \mathrm{MPa}$ at $25^{\circ} \mathrm{C}$ at $0.2 \mathrm{wt} \% \mathrm{MWCNT}$. This value is $20 \%$ and $13 \%$ higher than unloaded DGEDP-Bu and $0.2 \mathrm{wt} \%$ MWCNT DGEBA composites, respectively. These results indicate that in response to an oscillating strain, the filler network is more structurally supportive within DGEDP-Bu than DGEBA. The enhanced structural stability of the MWCNT networks in DGEDP-Bu is further demonstrated once the composites are subjected to temperatures $\geq \mathrm{T}_{\mathrm{g}}$. At the $\mathrm{T}_{\mathrm{g}}$ of composite, the matrix experiences a large reduction in elastic modulus and a peak of the $\tan \delta$ 
curve can be observed. At this temperature, polymer chain mobility is increased and the modulus of the reinforced composite gives way to moduli that predominantly depend on the percolating MWCNT network.[51] The relative storage modulus $\left(E^{\prime}{ }_{r e l}\right)$ for composites was calculated by normalizing $E$ ' of the composite with that of the respective neat epoxy at the same temperature $\left(E_{\text {rel }}{ }^{\prime}=E_{\text {composite }} / E_{r e s i n}\right)$. When the epoxies are in a glassy state at $25^{\circ} \mathrm{C}$, there is no notable variance between the relative storage moduli, however, at $30^{\circ} \mathrm{C}$ above $T_{g}$, DGEDP-Bu at $0.2 \mathrm{wt} \%$ exhibits a $240 \%$ increase over DGEBA at the same loading, and a 510\% increase over both neat DGEDP-Bu and DGEBA (Figure 6). This result is indicative of a more structurally stable and effective network of MWCNTs in the DGEDP-Bu in comparison with DGEBA.

Previous measurements of the molecular weight between crosslinks $\left(\mathrm{M}_{\mathrm{x}}{ }^{\mathrm{e}}\right)$ has indicated that DGEDP-Bu exhibits a $10 \%$ greater $\mathrm{M}_{\mathrm{x}}{ }^{\mathrm{e}}$ than DGEBA. [4] This result implies a decreased crosslink density of DGEDP-Bu with respect to DGEBA. Differences in crosslink density have been shown to alter the state of dispersion of MWCNTs in epoxy systems due to the interference of matrix crosslinking with filler cooperativity and an effective decrease in the length of cooperatively rearranging regions (CRR) when the crosslink density is too high.[52] The demonstrated lower crosslinking density of DGEDP-Bu may therefore allow for more interfacial mechanical interaction between particles in the matrix, improving the percolation behavior of the matrix and explaining the improvement seen in the $E_{r e l}$.

Table 5 lists composite $T_{\mathrm{g}}$ values as a function of MWCNT loading. No statistically significant differences were observed aside from the $T_{\mathrm{g}}$ of $0.2 \mathrm{wt} \%$ MWCNT DGEDP-Bu, which is $10^{\circ} \mathrm{C}$ lower than the $T_{\mathrm{g}}$ of unloaded DGEDP-Bu. Consistent with previous reports, unloaded DGEDP-Bu has an inherently lower $T_{\mathrm{g}}$ than DGEBA.[4] The lower $T_{\mathrm{g}}$ of DGEDP$\mathrm{Bu}$ is believed to result from the $n$-butyl-ester moieties that may act as an internal plasticizer.[4] We are currently investigating the effect of MWCNTs on curing kinetics which may affect the composite $T_{\mathrm{g}}$ at higher filler loadings.

\section{CONCLUSION}

The results of this work demonstrate that electrical, rheological, and mechanical percolation can be achieved in DGEDP$\mathrm{Bu}$, a biobased epoxy, at relatively low MWCNT loadings (0.05-0.1 wt \% MWCNT). This study stands as one of the first to fabricate biobased epoxy-CNT nanocomposites and provides evidence that DGEDP-Bu can function as a suitable replacement for DGEBA in a host of potential industries. Furthermore, discrepancies between percolation for various material parameters has been explored and described mechanistically.

DGEDP-Bu's superior percolation behavior supports a hypothesis of improved MWCNT network formation in DGEDP$\mathrm{Bu}$ over DGEBA due to differences in interfacial interaction with MWCNTs as well as variations in crosslinking density leading to enhanced filler cooperativity network mechanical structure. Incorporation of MWCNTs into a DGEDP-Bu matrix increased the elastic response of the matrix in rheological tests, achieved electrical conductivity, and improved dynamic 
mechanical properties while exhibiting varying property dependent percolation thresholds. The properties demonstrated by

DGEDP-Bu/MWCNT composites demonstrated excellent potential for use in fields relating to EMI/RF shielding,

microelectronics, structural composites, automotive, and aerospace industries.

\section{ACKNOWLEDGEMENTS}

The authors are grateful for funding received from the National Science Foundation Partnerships for International Research and Education (PIRE) Program (Award \#1243313).

\section{$\underline{\text { References }}$}

[1] S. Pillai, S. Ray, Epoxy-based carbon nanotubes reinforced composites, Adv. Nanocomposites - Synth. Charact. Ind. Appl. Ed. by Dr. Boreddy Reddy. (2011) 727. doi:10.5772/14915.

[2] R. Auvergne, S. Caillol, G. David, B. Boutevin, J.P. Pascault, Biobased thermosetting epoxy: Present and future, Chem. Rev. 114 (2014) 1082-1115. doi:10.1021/cr3001274.

[3] M. V Maffini, B.S. Rubin, C. Sonnenschein, A.M. Soto, Endocrine disruptors and reproductive health : The case of bisphenolA, 255 (2006) 179-186. doi:10.1016/j.mce.2006.04.033.

[4] A. Maiorana, S. Spinella, R.A. Gross, Bio-Based Alternative to the Diglycidyl Ether of Bisphenol A with Controlled Materials Properties, Biomacromolecules. 16 (2015) 1021-1031. doi:10.1021/acs.biomac.5b00014.

[5] Chemical Weekly, Challenging times for the bio-based chemical industry, (2015) 217-224.

[6] W. Bauhofer, J. Kovacs, A review and analysis of electrical percolation in carbon nanotube polymer composites, Compos. Sci. Technol. (2009) 1-26. doi:10.1016/j.compscitech.2008.06.018.A.

[7] C.A. Martin, J.K.W. Sandler, M.S.P. Shaffer, M.K. Schwarz, W. Bauhofer, K. Schulte, A.H. Windle, Formation of percolating networks in multi-wall carbon-nanotube-epoxy composites, Compos. Sci. Technol. 64 (2004) 2309-2316. doi:10.1016/j.compscitech.2004.01.025.

[8] J.Z. Kovacs, B.S. Velagala, K. Schulte, W. Bauhofer, Two percolation thresholds in carbon nanotube epoxy composites, 67 (2007) 922-928. doi:10.1016/j.compscitech.2006.02.037.

[9] S. Kirkpatrick, Percolation and Conduction, Rev. Mod. Phys. 45 (1973) 574-588. doi:10.1103/RevModPhys.45.574.

[10] D. Stauffer, a Aharony, Introduction to Percolation Theory, Computer (Long. Beach. Calif). 1 (1994) 192. doi:10.1103/RevModPhys.63.991.

[11] I. Alig, T. Skipa, D. Lellinger, M. Bierdel, H. Meyer, Dynamic percolation of carbon nanotube agglomerates in a polymer matrix: Comparison of different model approaches, Phys. Status Solidi Basic Res. 245 (2008) 2264-2267. doi:10.1002/pssb.200879622.

[12] S.H. Munson-Mcgee, Estimation of the critical concentration in an anisotropic percolation network, Phys. Rev. B. 43 (1991) 3331-3336. doi:10.1103/PhysRevB.43.3331.

[13] X. Zeng, X. Xu, P.M. Shenai, E. Kovalev, C. Baudot, N. Mathews, Y. Zhao, Characteristics of the electrical percolation in carbon nanotubes/polymer nanocomposites, J. Phys. Chem. C. 115 (2011) 21685-21690. doi:10.1021/jp207388n.

[14] C. Mcclory, T. Mcnally, M. Baxendale, P. Pötschke, W. Blau, M. Ruether, Electrical and rheological percolation of PMMA / MWCNT nanocomposites as a function of CNT geometry and functionality, Eur. Polym. J. 46 (2010) 854-868. doi:10.1016/j.eurpolymj.2010.02.009.

[15] J.K.W. Sandler, J.E. Kirk, I.A. Kinloch, M.S.P. Shaffer, A.H. Windle, Ultra-low electrical percolation threshold in carbonnanotube-epoxy composites, Polymer (Guildf). 44 (2003) 5893-5899. doi:10.1016/S0032-3861(03)00539-1.

[16] M. Martin-Gallego, M.M. Bernal, M. Hernandez, R. Verdejo, M.A. Lopez-Manchado, Comparison of filler percolation and mechanical properties in graphene and carbon nanotubes filled epoxy nanocomposites, Eur. Polym. J. 49 (2013) 1347-1353. doi:10.1016/j.eurpolymj.2013.02.033. 
[17] J. Coleman, S. Curran, a. Dalton, a. Davey, B. McCarthy, W. Blau, R. Barklie, Percolation-dominated conductivity in a conjugated-polymer-carbon-nanotube composite, Phys. Rev. B. 58 (1998) R7492-R7495. doi:10.1103/PhysRevB.58.R7492.

[18] G. Faiella, V. Antonucci, S.T. Buschhorn, L.A.S.A. Prado, K. Schulte, M. Giordano, Tailoring the electrical properties of MWCNT/epoxy composites controlling processing conditions, Compos. Part A Appl. Sci. Manuf. 43 (2012) $1441-1447$. doi:10.1016/j.compositesa.2012.04.002.

[19] a. Celzard, E. McRae, C. Deleuze, M. Dufort, G. Furdin, J. Marêché, Critical concentration in percolating systems containing a high-aspect-ratio filler, Phys. Rev. B. 53 (1996) 6209-6214. doi:10.1103/PhysRevB.53.6209.

[20] W. Bauhofer, S.C. Schulz, A.E. Eken, T. Skipa, D. Lellinger, I. Alig, E.J. Tozzi, D.J. Klingenberg, Shear-controlled electrical conductivity of carbon nanotubes networks suspended in low and high molecular weight liquids, Polymer (Guildf). 51 (2010) 5024-5027. doi:10.1016/j.polymer.2010.09.013.

[21] Y.Y. Huang, S. V Ahir, E.M. Terentjev, Dispersion rheology of carbon nanotubes in a polymer matrix, (2006) 1-9. doi:10.1103/PhysRevB.73.125422.

[22] J.J. Karippal, H.N.N. Murthy, K.S. Rai, M. Krishna, M. Sreejith, The Processing and Characterization of MWCNT/Epoxy and CB/Epoxy Nanocomposites Using Twin Screw Extrusion, Polym. Plast. Technol. Eng. 49 (2010) 1207-1213. doi:10.1080/03602559.2010.496413.

[23] S.C. Schulz, G. Faiella, S.T. Buschhorn, L.A.S.A. Prado, M. Giordano, K. Schulte, W. Bauhofer, Combined electrical and rheological properties of shear induced multiwall carbon nanotube agglomerates in epoxy suspensions, Eur. Polym. J. 47 (2011) 2069-2077. doi:10.1016/j.eurpolymj.2011.07.022.

[24] J. Sandler, M.S.. Shaffer, T. Prasse, W. Bauhofer, K. Schulte, a. . Windle, Development of a dispersion process for carbon nanotubes in an epoxy matrix and the resulting electrical properties, Polymer (Guildf). 40 (1999) 5967-5971. doi:10.1016/S0032-3861(99)00166-4.

[25] J.M. Brown, D.P. Anderson, R.S. Justice, K. Lafdi, M. Belfor, K.L. Strong, D.W. Schaefer, Hierarchical morphology of carbon single-walled nanotubes during sonication in an aliphatic diamine, Polymer (Guildf). 46 (2005) 10854-10865. doi:10.1016/j.polymer.2005.08.089.

[26] M. Chapartegui, N. Markaide, S. Florez, C. Elizetxea, M. Fernandez, A. Santamaría, Specific rheological and electrical features of carbon nanotube dispersions in an epoxy matrix, Compos. Sci. Technol. 70 (2010) 879-884. doi:10.1016/j.compscitech.2010.02.008.

[27] V. Fombuena, L. Bernardi, O. Fenollar, T. Boronat, R. Balart, Characterization of green composites from biobased epoxy matrices and bio-fillers derived from seashell wastes, Mater. Des. 57 (2014) 168-174. doi:10.1016/j.matdes.2013.12.032.

[28] R. Masoodi, R.F. El-Hajjar, K.M. Pillai, R. Sabo, Mechanical characterization of cellulose nanofiber and bio-based epoxy composite, Mater. Des. 36 (2012) 570-576. doi:10.1016/j.matdes.2011.11.042.

[29] H. Miyagawa, R.J. Jurek, A.K. Mohanty, M. Misra, L.T. Drzal, Biobased epoxy/clay nanocomposites as a new matrix for CFRP, Compos. Part A Appl. Sci. Manuf. 37 (2006) 54-62. doi:10.1016/j.compositesa.2005.05.014.

[30] L. Di Landro, G. Janszen, Composites with hemp reinforcement and bio-based epoxy matrix, Compos. Part B Eng. 67 (2014) 220-226. doi:10.1016/j.compositesb.2014.07.021.

[31] M.M. Bernal, M.A. Lopez-Manchado, R. Verdejo, In situ foaming evolution of flexible polyurethane foam nanocomposites, Macromol. Chem. Phys. 212 (2011) 971-979. doi:10.1002/macp.201000748.

[32] S.S. Rahatekar, K.K.K. Koziol, S. a. Butler, J. a. Elliott, M.S.P. Shaffer, M.R. Mackley, a. H. Windle, Optical microstructure and viscosity enhancement for an epoxy resin matrix containing multiwall carbon nanotubes, J. Rheol. (N. Y. N. Y). 50 (2006) 599. doi:10.1122/1.2221699.

[33] N. Casson, Mill CC Ed.. Rheology of Disperse Systems, (1959).

[34] M.K. Tiwari, A. V. Bazilevsky, A.L. Yarin, C.M. Megaridis, Elongational and shear rheology of carbon nanotube suspensions, Rheol. Acta. 48 (2009) 597-609. doi:10.1007/s00397-009-0354-z.

[35] H. Xia, M. Song, Preparation and characterization of polyurethane-carbon nanotube composites, Soft Matter. 1 (2005) 386. doi:10.1039/b509038e.

[36] P.E.D. Augusto, M. Cristianini, A. Ibarz, Effect of temperature on dynamic and steady-state shear rheological properties of siriguela (Spondias purpurea L.) pulp, J. Food Eng. 108 (2012) 283-289. doi:10.1016/j.jfoodeng.2011.08.015.

[37] I.A. Kinloch, S.A. Roberts, A.H. Windle, A rheological study of concentrated aqueous nanotube dispersions, Polymer (Guildf). 43 (2002) 7483-7491. doi:10.1016/S0032-3861(02)00664-X.

[38] M. Abdalla, D. Dean, D. Adibempe, E. Nyairo, P. Robinson, G. Thompson, The effect of interfacial chemistry on molecular mobility and morphology of multiwalled carbon nanotubes epoxy nanocomposite, Polymer (Guildf). 48 (2007) 5662-5670. 
doi:10.1016/j.polymer.2007.06.073.

[39] Y.T. Wang, C.S. Wang, H.Y. Yin, L.L. Wang, H.F. Xie, R.S. Cheng, Carboxyl-terminated butadiene-acrylonitrile-toughened epoxy/carboxyl-modified carbon nanotube nanocomposites: Thermal and mechanical properties, Express Polym. Lett. 6 (2012) 719-728. doi:10.3144/expresspolymlett.2012.77.

[40] R. Le Bouc, P. Lenfant, X. Delbeuck, L. Ravasi, F. Lebert, F. Semah, F. Pasquier, My belief or yours? Differential theory of mind deficits in frontotemporal dementia and Alzheimer's disease., Brain. 135 (2012) 3026-38. doi:10.1093/brain/aws237.

[41] M. Yoonessi, M. Lebrón-Colón, D. Scheiman, M.A. Meador, Carbon Nanotube Epoxy Nanocomposites: The Effects of Interfacial Modifications on the Dynamic Mechanical Properties of the Nanocomposites, ACS Appl. Mater. Interfaces. 6 (2014) 16621-16630. doi:10.1021/am5056849.

[42] V. Mittal, Polymer Nanotube Nanocomposites: Synthesis, Properties, and Applications, Wiley, 2010. https://books.google.com/books?id=_kbyzcV_zBoC.

[43] X.L.Z. A. H. KORAYEM, S. CHUAH, G. P. SIMON, W.H. DUAN, A Comparative Study on Epoxy Nanocomposites Reinforced By Carbon Nanotubes : Effect of Fabrication Methods, Hokkaido Univ. Collect. Sch. Acad. Pap. (2013). http://hdl.handle.net/2115/54293.

[44] A. Haesch, T. Clarkson, J. Ivens, S. V. Lomov, I. Verpoest, L. Gorbatikh, Localization of carbon nanotubes in resin rich zones of a woven composite linked to the dispersion state, Nanocomposites. 1 (2015) 204-213. doi:10.1080/20550324.2015.1117306.

[45] M.R. Loos, J. Yang, D.L. Feke, I. Manas-Zloczower, R. Sengupta, S. Chakraborty, S. Bandyopadhyay, S. Dasgupta, R. Mukhopadhyay, K. Auddy, a S. Deuri, Enhanced fatigue life of carbon nanotube-reinforced epoxy composites, Polym. Eng. Sci. 52 (2012) 1882-1887. doi:10.1002/pen.23145.

[46] E.E. Groop, a W. Nowicki, C.I. Calle, C.R. Buhler, J.G. Mantovani, Comparison of Surface Resistivity and Triboelectric Charge Generation Characteristics of Materials, Proc. 40th Sp. Congr. (2003).

http://scholar.google.ca/scholar?q=Comparison+of+Surface+Resistivity+and+Triboelectric+Charge+Generation\&hl=en\&as_s $\mathrm{dt}=0 \&$ as_vis=1\&oi=scholart\&sa=X\&ei=tW5VVdnPKYOUyAS77oHwBQ\&ved=0CBoQgQMwAA.

[47] S. Cui, R. Canet, A. Derre, M. Couzi, P. Delhaes, Characterization of multiwall carbon nanotubes and influence of surfactant in the nanocomposite processing, Carbon N. Y. 41 (2003) 797-809. doi:10.1016/S0008-6223(02)00405-0.

[48] A. Allaoui, S. Bai, H.M. Cheng, J.B. Bai, Mechanical and electrical properties of a MWNT/epoxy composite, Compos. Sci. Technol. 62 (2002) 1993-1998. doi:10.1016/S0266-3538(02)00129-X.

[49] S.-M. Yuen, C.-C.M. Ma, H.-H. Wu, H.-C. Kuan, W.-J. Chen, S.-H. Liao, C.-W. Hsu, H.-L. Wu, Preparation and Thermal, Electrical, and Morphological Properties of Multiwalled Carbon Nanotube and Epoxy Composites, J. Appl. Polym. Sci. 103 (2006) 1272-1278. doi:10.1002/app.

[50] L. Liu, S. Matitsine, Y.B. Gan, L.F. Chen, L.B. Kong, K.N. Rozanov, Frequency dependence of effective permittivity of carbon nanotube composites, in: J. Appl. Phys., 2007. doi:10.1063/1.2728765.

[51] R. Kotsilkova, D. Fragiadakis, P. Pissis, Reinforcement effect of carbon nanofillers in an epoxy resin system: Rheology, molecular dynamics, and mechanical studies, J. Polym. Sci. Part B Polym. Phys. 43 (2005) 522-533. doi:10.1002/polb.20352.

[52] K.W. Putz, M.J. Palmeri, R.B. Cohn, R. Andrews, L.C. Brinson, Effect of Cross-Link Density on Interphase Creation in Polymer Nanocomposites Effect of Cross-Link Density on Interphase Creation in Polymer Nanocomposites, Society. (2008) 6752-6756. doi:10.1021/ma800830p. 